Revista Destaques Acadêmicos, Lajeado, v. 12, n. 4, 2020. ISSN 2176-3070

DOI: http://dx.doi.org/10.22410/issn.2176-3070.v12i4a2020.2758

http://www.univates.br/revistas

\title{
BLUEPATH: SISTEMA DE LOCALIZAÇÃO INDOOR
}

\author{
Raul Silvério Mittelstadt ${ }^{1}$, Fabrício Pretto ${ }^{2}$
}

Resumo: Localizar-se atualmente tornou-se um desafio a ser enfrentado. Com o desenvolvimento das cidades, a quantidade de rotas e lugares aumentou consideravelmente. Para suprir essa necessidade de chegar em lugares desconhecidos ou a melhor rota a seguir, surgiu o GPS que se tornou popular rapidamente, devido à sua grande efetividade e quantidade de ferramentas. No entanto, o GPS tem suas limitações no que diz respeito à precisão, o que inviabiliza sua utilização em ambientes internos. Neste sentido este trabalho apresenta o desenvolvimento de um aplicativo de localização indoor, para facilitar o processo de localização em ambientes fechados. Para atingir tal objetivo, foram distribuídos transmissores Bluetooth em um ambiente interno para que o aplicativo consiga, por meio dos transmissores, determinar a localização. Para determinar a localização, foram utilizadas técnicas de orientação como: RSSI (Received Signal Strength Indicator) e ToA (Time of Arrival). Um estudo detalhado das tecnologias e técnicas envolvidas, contemplam o escopo deste trabalho. Com os resultados obtidos foi possível determinar a localização do smartphone em um ambiente interno.

Palavras-chave: Localização indoor, GPS, Tecnologia, Técnicas

\section{INTRODUÇÃO}

Com o desenvolvimento da humanidade, a localização se tornou uma preocupação constante, as razões podem ser as mais diversas como econômicas ou pessoais. A tecnologia que mais se popularizou na questão de localização foi o GPS. Essa tecnologia revolucionou a área de localização, possibilitando a criação de diversos aplicativos com as mais variadas serventias, como localização de pessoas e veículos, recursos de jogos, entre outros. Segundo Soares (2015), existem alguns cenários que o GPS não pode atuar por não haver uma precisão aceitável. Ele relata que em lugares fechados ou em lugares subterrâneos o GPS

1 Engenheiro da Computação - Univates - rsmittelstadt@universo.univates.br

2 Mestre em Ciência da Computação - Univates - fabricio.pretto@univates.br 
não funciona corretamente, e para estes cenários mencionados devemos buscar alternativas de localização.

Mas o desafio não é somente se localizar em ambiente abertos, mas também em ambientes fechados, como andares de prédios, túneis, salas ou auditórios. Nestes espaços, o uso do GPS não é eficiente, por esse motivo foi necessário investir em novas tecnologias que pudessem atender essa demanda. Segundo Filho (2016), foram desenvolvidas técnicas de posicionamento indoor que utilizavam tecnologias sem fio já existentes no mercado, como Wireless, RFID, Infravermelho e Bluetooth. Algumas das técnicas utilizadas para localização indoor são ToA (Time of Arrival), TDoA (Time Difference of Arrival), AoA (Angle of Arrival) e RSS (Received Signal Strength).

Conforme o entendimento de Reck (2016), na atualidade existem muitas aplicações que necessitam da posição exata do aparelho, inclusive em ambientes internos. Para prover melhor localização interna, existem algumas tecnologias que podem ser utilizadas, em particular Bluetooth 4.0 se destaca. $\mathrm{O}$ autor explica que por este dispositivo consumir pouca energia e haver vários aparelhos novos saindo de fábrica com compatibilidade, por esse motivo essa tecnologia se torna muito atrativa e vantajosa. A Figura 1 mostra o crescimento das tecnologias sem fio dos últimos 20 anos, as áreas retangulares que estão na horizontal retratam: desenvolvimento, lançamento e estabilização.

O objetivo do presente trabalho consiste em criar uma solução em geolocalização em ambientes indoor utilizando tecnologias já existentes no mercado, que possibilite determinar a localização em ambientes fechados por meio da tecnologia de Bluetooth.

\section{REFERENCIAL TEÓRICO}

Neste capítulo é apresentada uma revisão das características e tecnologias que são utilizadas para o tema do trabalho desenvolvido.

\subsection{Categorias de Sistemas de Posicionamento}

Os sistemas de posicionamento podem ser divididos em 4 tipos, são eles: GPS, Infravermelho, Rádio e Ultrassom. Para localização em ambientes externos se adotou como padrão o GPS, pois foi um sistema bem-sucedido. No entanto, o GPS não funciona da mesma maneira em ambientes fechados, pois sua precisão pode não ser exata, fazendo com que esta tecnologia seja ineficaz para este tipo de aplicação.

Segundo a compreensão Farid, Nordin e Ismail (2013) uma tecnologia que pode ser utilizada em ambientes internos é o infravermelho. Ela pode ser utilizada para detecção de objetos que são estáticos ou móveis. Essa tecnologia está embutida em vários dispositivos móveis, TVs e sua utilização é relativamente fácil. A desvantagem são questões como a limitação em determinar a localização, privacidade e segurança. $\mathrm{O}$ infravermelho ainda 
sofre muito com interferência da luz de lâmpadas assim como a luz solar. Seu hardware também tem um alto investimento, o que torna a tecnologia menos atraente para essa aplicação.

As tecnologias baseadas em ultrassom não são fáceis de implementar pois as ondas de som geralmente sofrem reflexão em objetos que estão dispostos no ambiente, o que torna complicado a determinação da posição.

\subsection{Localização Interna}

As tecnologias de localização interna (indoor), geralmente não utilizam fios, para prover uma maior aceitação pelos usuários, já que não seria conveniente sair andando com cabos para ter que se localizar em algum ambiente. Assim as tecnologias sem fios garantem mais conforto, comodidade e segurança. Existem algumas maneiras neste sentido para prover a localização em ambientes internos, em seguida algumas delas serão explicadas.

\subsubsection{Wi-Fi}

O Wireless Fidelity chamado geralmente de Wi-Fi, é usado para se referir a redes que trabalham em especificações IEEE 802.11. Esse padrão foi lançado em 1997 e proporcionava taxas de 1 a 2 Mbps utilizando a frequência de $2.4 \mathrm{MHz}$. Esse padrão opera em frequências de ISM (Industrial, Scientific and Medical), ou seja, não precisa estar licenciado para funcionar.

Filho (2016) menciona que um fato que levou a tecnologia a ser aceita pelos usuários em localização indoor, é a grande quantidade de dispositivos que transmitem este sinal, tanto em lugares públicos como em privados. Mas para conseguir criar um sistema de localização indoor com Wi-Fi mais preciso, utiliza-se a planta baixa do local. Assim pode-se reduzir a distorção do sinal $\mathrm{Wi}-\mathrm{Fi}$, pelos materiais que podem existir no ambiente como eletrodomésticos, paredes ou obstáculos.

\subsubsection{RFID}

Segundo Abreu (2014) o RFID (Radio-Frequency Identification, em português Identificação de Rádio Frequência) é utilizada geralmente em identificação de produtos e rastreamento de inventário entre outros. Ele permite fazer a recuperação de dados através de uma transmissão eletromagnética com algum circuito que tenha sido integrado a ele.

Abreu (2016) relata que o RFID funciona com alguns componentes que incluem etiquetas que são afixados nas embalagens, leitores e a comunicação entre eles. O leitor de RFID lê os dados que são emitidos pelas etiquetas, elas devem estar na mesma frequência de rádio e protocolo para fazer a transmissão e recebimentos dos dados. Alguns itens contendo RFID estão sendo mostrados na Figura 10. 
Conforme o entendimento de Dalfovo, Hostins (2010) etiquetas podem ser caracterizadas como ativas e passivas, sendo que as etiquetas passivas trabalham sem baterias. Elas funcionam refletindo o sinal que foi transmitido pelo leitor adicionando informação, modulando o sinal que foi refletido. As etiquetas ativas são utilizadas para a substituição do código de barras tradicional. A diferença principal entre a ativa e passiva é o seu alcance. A passiva pode chegar a uma distância de 100 metros com uma velocidade de menos de 100 milissegundos.

\subsubsection{Bluetooth}

Segundo Billo (2003) o Bluetooth foi desenvolvido em 1994 pela empresa Ericsson, com o objetivo de fazer dispositivos se comunicarem sem a utilização de cabos. Para isso, a empresa desenvolveu uma comunicação com sinais de rádio que não fossem caros para este fim. A primeira criação de um produto foi chamada de Mclink, que tinha um curto alcance, utilizando baixa potência para não consumir tanta energia. O produto se tornou tão promissor que em 1997 atraiu a atenção de outras empresas para a comunicação sem fio. Billo (2003) relata que em 1998 foi criado o Bluetooth SIG (Special Interest Group), onde participaram grandes empresas como Intel, Ericsson, IBM, Toshiba e Nokia. Com todas estas empresas trabalhando juntas, a tecnologia se aperfeiçoou ao ponto de ser utilizada em vários aparelhos além de celulares.

Reck (2016) afirma que a tecnologia Bluetooth evoluiu e se popularizou entre os mais diversos dispositivos do mercado. Atualmente ele trabalha em frequência não licenciada entre 2,4 GHz e 2,485 GHz, sendo que sua grande vantagem é de transmitir dados e voz, ambos simultaneamente. Para trabalhar em banda não licenciada, o Bluetooth utiliza-se de um recurso chamado $\mathrm{AFH}$ (Adaptive Frequency Hopping, em português Saltos Adaptativos de Frequência) que corresponde a ação de dar saltos adaptativos entre as frequências para a diminuição das interferências entre aparelhos que utilizam a faixa de $2.4 \mathrm{GHz}$.

Com a evolução dos dispositivos que continham o Bluetooth, algumas categorias foram criadas para atender as mais diversas funcionalidades. Cada categoria tem sua distância e a potência máxima descrita na Quadro 1.

Um Bluetooth de classe 3 pode se conectar a um de classe 2 ou 1, no entanto eles conseguirão se comunicar somente se a distância do menor for respeitada. Isso significa que dispositivos que utilizam a classe 3 podem ser utilizados com classes inferiores sem problemas, contanto que suas limitações sejam respeitadas.

As conexões Bluetooth podem ser feitas de duas formas diferentes, elas podem ser assíncronas ou síncronas. Em ambos os casos, quem decide qual tipo será feito é sempre o master.

Martincoski (2003) afirma que a conexão Bluetooth assíncrona é conhecida como ACL (Asynchronous Connection-Less, em português Conexão 
Assíncrona), sua principal função é assegurar a integridade dos dados que estão sendo transmitidos. São feitas verificações de erros e retransmissões de pacotes caso tenha ocorrido algum problema. Um dispositivo Master pode fazer uma conexão ACL com vários Slaves, no entanto, o Slave pode manter somente uma conexão ACL. Já a conexão síncrona é conhecida como SCO (Synchronous Connection-Oriented, em português Conexões Síncronas), elas realizam transmissões regulares dos dados em uma taxa igual nos dois sentidos, sendo que os pacotes jamais são retransmitidos, ou seja, os dados transmitidos podem não estar íntegros. A conexão SCO pode ser estabelecida somente depois de uma conexão ACL já tenha sido estabelecida previamente, sendo que um dispositivo Master pode conectar-se somente com três Slaves simultaneamente. A principal aplicação para este tipo de conexão são dados de áudio, pois caso perca alguma informação no meio da transmissão, não há a necessidade de recuperá-lo.

\section{METODOLOGIA}

A metodologia de pesquisa deste trabalho enquanto seus procedimentos técnicos foi a pesquisa bibliográfica e experimental. Gil (2008) destaca que a pesquisa bibliográfica é desenvolvida a partir de um material já elaborado, que é constituído principalmente de livros e artigos.

O sistema é composto com 2 componentes essenciais, os transmissores e receptores. Para receptor, foi utilizado um smartphones, que receberá o sinal dos transmissores e posteriormente verificará a posição atual do dispositivo.

Para fazer a transmissão do sinal Bluetooth, utilizou-se o módulo NodeMCU ESP32. Ele é um módulo focado principalmente em robótica e automação, sendo voltado principalmente para IoT (Internet das coisas, em inglês Internet of Things). Segundo Aita (2017), o NodeMCU ESP32 é um microcontrolador que é utilizado em casos específicos, que não exijam grande quantidade de dados, pois estes dispositivos não contêm grandes quantidades de memória de armazenamento.

\section{EXPERIMENTAÇÃO}

O aplicativo visa determinar a sua localização dentro de um ambiente interno em tempo real, sem a necessidade de conhecimento prévio do local. Emissores de sinal Bluetooth foram distribuídos e afixados no local, para que o dispositivo consiga captar o sinal e aplicar a técnica de Triangulação (latência). Em seguida o dispositivo mostra a posição estimada que foi calculada.

\subsection{Ambiente}

O ambiente escolhido para realização da validação do experimento foi um corredor do Prédio 11 da Universidade do Vale do Taquari - 
Univates, em Lajeado - RS. A área total do local é aproximadamente $119 \mathrm{~m}^{2}$ (metros quadrados). A Figura 2 apresenta o layout do corredor e onde foram posicionados os emissores.

\subsection{Requisitos do sistema}

O aplicativo Android foi desenvolvido para calcular a distância estimada do smartphone dentro do ambiente, no entanto ele necessita de 4 processos para que isso aconteça, são eles:

1. O Bluetooth do smartphone precisa estar ativado, para isso existe um botão para ligar e desligar o Bluetooth.

1. O Bluetooth necessita estar visível para que ele possa encontrar os emissores.

2. O smartphone após os passos anteriores precisa começar a procurar emissores, para que possa pegar a intensidade do sinal e fazer os cálculos necessários.

3. Para fazer o cálculo, o smartphone necessita de 3 emissores ao seu alcance

\subsection{Emissores}

Para a emissão do sinal foram utilizados cinco módulos NodeMCU ESP32, eles já contemplam o Bluetooth instalado na placa, não sendo preciso instalar qualquer tipo de módulo extra.

Para que os nodes emitissem o sinal Bluetooth pelo ambiente, a configuração utilizada foi retirada da Espressif, que é a fabricante destes equipamentos. O exemplo que se utilizou foi o "BLE_server". O código cria um servidor localmente, assim, possibilitando que outros equipamentos possam enxergá-lo. Algumas informações como o UUID (Universally Unique Identifier) e o nome do dispositivo são colocados junto em sua programação. O UUID é de extrema importância, pois posteriormente na aplicação do smartphone esse parâmetro é utilizado para saber de quem está vindo o sinal, e sendo assim, saber a sua posição do emissor no ambiente.

\subsection{Aplicativo Android}

Para o execução do aplicativo 3 requisitos devem observados, sendo eles o do Bluetooth estar ativo, o Bluetooth estar visível e posteriormente a descoberta dos emissores, obtendo a intensidade do sinal de cada um. Seguindo os requisitos, a interface do aplicativo foi desenvolvida com 3 botões na parte superior do aplicativo, para ligar e desligar o Bluetooth do dispositivo, habilitar a visibilidade do dispositivo e a descoberta dos emissores. Após a descoberta de 3 emissores e fazer os cálculos o resultado é mostrado no centro da tela, informando a posição estimada do dispositivo, como mostra a Figura 3. 
Para que a posição pudesse ser estimada foi utilizada a técnica de triangulação, onde foi necessário ter cobertura de 3 emissores. Se houver um número menor que isso a técnica não consegue estimar a posição pois geram pontos imprecisos. Após a detecção de 3 emissores, o primeiro passo é determinar a distância de cada um deles. A fórmula utilizada neste caso foi:

$\mathrm{D}=\mathrm{A} *(\mathrm{r} / \mathrm{t}) \wedge \mathrm{B}+\mathrm{C}$

- $\mathrm{D}=$ Distância estimada

- $r=E$ a medida do RSSI medido do dispositivo

- $\mathrm{t}=$ É a medida de referência à 1 metro do emissor

- A, B, C = São constantes da fórmula e dependem diretamente da medida de 1 metro como referência

Para a obtenção das constantes da fórmula, foram feitas as medidas do RSSI nas distâncias de 0,25, 0.5, 1, 2, 3, 4, 5, 6, 7, 8, 9, 10, 11 e 12 metros, a medida do RSSI de referência que foi obtido a um metro foi de -64db. De posse dos valores das distâncias e seus respectivos RSSI, coloca-se em uma coluna os valores do sinal medido no smartphone, dividido pelo sinal base medido a 1 metro de distância. Na outra coluna temos os valores das distâncias de 0,25 até 12 metros. Para a determinação de A e B se faz uma relação entre as duas colunas utilizando a técnica de regressão que permite explorar a relação entre as duas colunas. Esse processo se denomina regressão de potência.

Os valores de A e B obtidos foram 0,7945250143 e 7,171541075 respectivamente. Agora com as duas constantes iniciais já foi possível obter a distância estimada, e para obter a constante $C$, o valor da distância real medido a um metro foi subtraído da distância estimada também a um metro, o resultado obtido foi 0,2054749857 . O método que é executado dentro da aplicação ficou ilustrado na Figura 23.

Com a distância estimada de cada emissor, é possível aplicar o método da triangulação. $\mathrm{O}$ este método necessita receber a informação de onde o emissor está localizado, então é passado uma posição x e y da posição de cada um deles, juntamente a distância que o smartphone estava do emissor. O método que faz a triangulação dentro do aplicativo e retorna a posição estimada final.

\subsection{Testes}

Para a realização dos testes, foram testados em 8 pontos diferentes do ambiente e para cada teste foram feitas 3 tentativas. A posição certa onde foram realizados os testes estão ilustrados na Figura 4.

Para cada ponto foram feitas 3 tentativas onde foram obtidos diferentes resultados de localização. Para saber o quão preciso foi o sistema, calculou-se o erro que ocorreu em cada uma das tentativas, então em uma coluna está o erro obtido no eixo $\mathrm{X}$ e outra coluna está o erro obtido no eixo $\mathrm{Y}$. O erro calculado 
é em relação a posição real, e os dados podem ser conferidos em metros na Tabela 1.

Tabela 1: Resultados dos Pontos

\begin{tabular}{c|c|c|c|c|c|c|c}
\hline Ponto & Tentativa & $\begin{array}{c}\text { Distância } \\
\text { Real X }\end{array}$ & $\begin{array}{c}\text { Distância } \\
\text { Real Y }\end{array}$ & $\begin{array}{c}\text { Distância } \\
\text { estimada X }\end{array}$ & $\begin{array}{c}\text { Distância } \\
\text { estimada Y }\end{array}$ & $\begin{array}{c}\text { Erro } \\
\text { em X }\end{array}$ & $\begin{array}{c}\text { Erro } \\
\text { em Y }\end{array}$ \\
\hline 1 & 1 & 1 & 4 & 1,54 & 5,3 & 0,54 & 1,3 \\
\hline 1 & 2 & 1 & 4 & 1,7 & 4,3 & 0,7 & 0,3 \\
\hline 1 & 3 & 1 & 4 & 0,9 & 4,5 & $-0,1$ & 0,5 \\
\hline 2 & 1 & 2,5 & 6 & 3,1 & 5,4 & 0,6 & $-0,6$ \\
\hline 2 & 2 & 2,5 & 6 & 2,3 & 5,2 & $-0,2$ & $-0,8$ \\
\hline 2 & 3 & 2,5 & 6 & 2 & 6,5 & $-0,5$ & 0,5 \\
\hline 3 & 1 & 2,5 & 13 & 2,3 & 10 & $-0,2$ & -3 \\
\hline 3 & 2 & 2,5 & 13 & 2 & 12,3 & $-0,5$ & $-0,7$ \\
\hline 3 & 3 & 2,5 & 13 & 3,5 & 11,5 & 1 & $-1,5$ \\
\hline 4 & 1 & 6 & 5 & 6,8 & 5,3 & 0,8 & 0,3 \\
\hline 4 & 2 & 6 & 5 & 5,6 & 4,5 & $-0,4$ & $-0,5$ \\
\hline 4 & 3 & 6 & 5 & 6,3 & 4 & 0,3 & -1 \\
\hline 5 & 1 & 6 & 1 & 5,3 & 1,5 & $-0,7$ & 0,5 \\
\hline 5 & 2 & 6 & 1 & 5 & 1,6 & -1 & 0,6 \\
\hline 5 & 3 & 6 & 1 & 4,7 & 0,6 & $-1,3$ & $-0,4$ \\
\hline 6 & 1 & 15 & 6 & 13 & 5 & -2 & -1 \\
\hline 6 & 2 & 15 & 6 & 12,7 & 6,8 & $-2,3$ & 0,8 \\
\hline 6 & 3 & 15 & 6 & 14,5 & 4,3 & $-0,5$ & $-1,7$ \\
\hline 7 & 1 & 22 & 4,5 & 25 & 6 & 3 & 1,5 \\
\hline 7 & 2 & 22 & 4,5 & 24,3 & 5 & 2,3 & 0,5 \\
\hline 7 & 3 & 22 & 4,5 & 20 & 3,9 & -2 & $-0,6$ \\
\hline 8 & 1 & 8,5 & 3,5 & 8 & 4 & $-0,5$ & 0,5 \\
\hline 8 & 2 & 8,5 & 3,5 & 7,5 & 3 & -1 & $-0,5$ \\
\hline 8 & 3 & 8,5 & 3,5 & 8,9 & 2,7 & 0,4 & $-0,8$ \\
\hline
\end{tabular}

Fonte: Do Autor (2018).

Os resultados encontrados nos pontos 1,2,4,5e 8 foram mais satisfatórios, pois eles contêm uma baixa taxa de erro variando um metro para mais ou para menos. Esses resultados foram obtidos pois os pontos estão localizados em um local mais central, onde o smartphone fica no centro, sendo assim, mais fácil de ter uma medida precisa e de fazer do cálculo de triangulação corretamente.

$\mathrm{O}$ resultado do ponto 3 teve uma margem de erro maior em relação aos pontos anteriores, onde ela chegou a variar até três metros de distância em uma das tentativas, mas nas outras tentativas acabou ficando um pouco acima de um metro de erro. Essa variação deve-se ao fato de este ponto estar em uma das extremidades do corredor, onde não é possível que o smartphone fique cercado por emissores. 
Já os pontos 6 e 7 tiveram os piores resultados, podendo variar de dois a três metros para mais ou para menos. Esses pontos ficam com a margem de erro maior pois estão no corredor e não conseguem estar cercado pelos emissores.

Analisando os dados obtidos, percebe-se que quanto mais cercado estiver de emissores e mais perto eles estiverem, maior é a precisão, Isso ocorre por dois motivos. O primeiro é que o RSSI obtido é mais intenso e varia menos quanto mais perto do emissor ele se encontrar. Essa característica garante que quanto mais perto dos emissores melhores e mais confiáveis serão os dados. O segundo motivo é o método da triangulação, para que ele funcione melhor, quanto mais espalhados em relação ao smartphone eles estiverem melhor.

Os resultados não são mais expressivos pois existem algumas características que influenciam diretamente no resultado e na confiabilidade dos dados, mas a principal certamente é a interferência, que influência na captação do sinal, gerando ruido na hora da leitura. Outro ponto a se considerar é o ambiente, onde há muitas paredes e que os emissores não ficam totalmente à vista por estarem em um corredor diferente, o sinal também é afetado. Por esse motivo os emissores ficaram na posição vertical, pois o componente responsável por emitir o sinal Bluetooth fica na parte de cima do emissor. Então quando colocado na posição vertical, o sinal mais intenso se espalha pelo ambiente, se ficasse na posição horizontal ele emitiria sinal mais intensamente para o teto.

\section{CONSIDERAÇÕES FINAIS}

A localização indoor é uma área que vem sendo estudada e aperfeiçoada nos últimos anos, e se mostra uma tecnologia que estará cada vez mais presente no dia a dia. A quantidade de aplicações que pode ser desenvolvida neste setor ainda é bastante grande devido a sua versatilidade.

Esse trabalho apresentou algumas técnicas para a localização indoor, mas a escolhida para ser implementada foi a triangulação. Essa técnica se destaca pela sua precisão, pois nos testes realizados foi possível obter precisões que variavam de um a três metros. Outras abordagens também poderiam ser aplicadas dependendo de cada situação.

O trabalho tinha como objetivo principal, desenvolver uma aplicação de custo acessível, para a localização em ambientes internos. Outros propósitos eram montar o hardware para fazer a emissão do Bluetooth, documentar técnicas e testar a solução em laboratórios. Todas esses objetivos foram cumpridos com êxito no decorrer da atividade.

Para trabalhos futuros e o aprimoramento da aplicação, seria interessante a adição de uma ou mais técnicas de localização ao aplicativo, para que haja mais precisão. Para aumentar a confiabilidade dos dados poderiam ser utilizados outros sinais, como por exemplo a rede wireless. Todas essas técnicas e adição de elementos, poderiam melhorar ainda mais a precisão e a confiabilidade dos dados obtidos. 


\section{REFERÊNCIAS}

ABREU, Rafael Fernando Pereira - Localização Indoor em Ambientes Inteligentes Universidade do Minho, 2014

AITA, Ricardo Hahn - Sistema de irrigação localizada e automatizada, Pontifícia Universidade católica do Rio Grande do Sul, 2017

BILLO, Eduardo Afonso - Uma pilha de protocolos Bluetooth adaptável a aplicação - Universidade Federal de Santa Catarina, 2003

DALFOVO, Oscar; Hostins, Clovis Anderson - Delineamento para aplicação do RFID na logística de supermercado como inteligência competitiva : Supermercado Hostins, 2010

FARID, Zahid; NORDIN, Rosdiadee; Ismail, Mohamod - Recent Advances in Wireless Indoor Localization Techniques and System, 2013.

FILHO, Francisco Lopes Daniel - Uma API para detecção de presença de dispositivos móveis em ambientes Indoor - Universidade Federal Do Ceará, 2016

GIL, Antonio Carlos - Métodos e técnicas de pesquisa social - 6 ed São Paulo: atlas 2008

GU, Yanying; LO, Anthony; NIEMEGEERS, Ignas - A Survey of Indoor Positioning Systems for Wireless Personal Networks, 2009

HIGHTOWER, Jeffrey; BORRIELLO, Gaetano - Location Sensing Techniques University of Washington, 2001

JUNIOR, Valmir Antonio Schneider; ANDREÃO, Rodrigo Varejão; NUNES, Reginaldo Barbosa - Sistema de localização para ambientes fechados baseado na intensidade de sinal recebido em rede ZigBee - Universidade Federal do Espirito Santo, 2011

KOSKIOLA, Annina; Indoor Navigation in Complex buildings - exclusive sneak peek. proximi.io, 2017. Disponível em: <https:/ / proximi.io/indoor-navigation-complexbuildings/>. Acesso em: 29 maio 2018

LAKATOS, Eva Maria; MARCONI, Maria de Andrade - Fundamentos de metodologia científica - 7 ed São Paulo: atlas 2010

LAKATOS, Eva Maria; MARCONI, Maria de Andrade - Técnicas de Pesquisa- 8 ed São Paulo: atlas 2018

MARTINCOSKI, Daniel Henrique - Sistema para telemetria de eletrocardiograma utilizando tecnoligia Bluetooth - Universidade Federal de Santa Catarina, 2003 MOURA, André Iasi - WBLS: Um sistema de localização de dispositivos móveis em redes Wi-Fi, Universidade de São Paulo, 2007. 
RECK, Arcelo Sala - Beacons BLE - BLUETOOTH LOW ENERGY - Design e análise de um sistema de localização indoor Universidade de Caxias do Sul, 2016.

SOARES, Fábio Miguel Ramos - Localização baseada em medidas de distância para sistemas interiores de larga escala, Engenharia Eletrotécnica e de Computadores, 2015. 